\title{
Analysis of Diastolic Left Ventricular Function in Adolescents with Juvenile Systemic Lupus Erythematosus
}

Talita Nolasco Loureiro, ${ }^{\circledR}$ Cristina Ortiz Sobrinho Valete, ${ }^{2}{ }^{\circledR}$ Marcia Bueno Castier, ${ }^{\circledR}$ Maria de Fatima Monteiro Leite, ${ }^{3}$ Flavio Roberto Sztajnbok ${ }^{1 \oplus}$

Pós-graduação em Ciências Médicas - Universidade do Estado do Rio de Janeiro, ${ }^{1}$ Rio de Janeiro, RJ - Brazil

Área de Saúde da Criança e do Adolescente, Departamento de Medicina, Universidade Federal de São Carlos, ${ }^{2}$ São Paulo, SP - Brazil

Instituto Fernandes Figueira - FIOCRUZ, ${ }^{3}$ Rio de Janeiro, RJ - Brazil

\section{Abstract}

Background: Juvenile systemic lupus erythematosus (JSLE) is a chronic inflammatory disease that affects the heart in $50 \%$ of cases. The behavior of diastolic function in adolescents and the predictors of its occurrence by conventional echocardiography are poorly established.

Objectives: This study aimed to evaluate diastolic function in adolescents with JSLE and to identify possible predictors of its occurrence by conventional echocardiography.

Methods: Cross-sectional, observational, control group study in a tertiary hospital of 49 adolescents with JSLE and 49 controls, using the EACVI 2016 guideline classification. Statistical methods used were Fisher and Mann-Whitney tests. Multivariate logistic regression models were constructed. A significance level of $5 \%$ was adopted.

Results: Among 98 patients, the JSLE group had higher indexed left atrial volume ( $p<0.001)$, lower lateral E' value $(p<0.001)$ and lower E/A ratio value $(p<0.001)$. The diagnosis of JSLE was associated with a higher chance of increased left atrial index volume (OR 3.3; $\mathrm{p}$ value 0.03 ).

Conclusions: Based on the 2016 guideline, no diastolic dysfunction was found in JSLE. However, differences in the analyzed echocardiographic parameters were found in these adolescents.

Keywords: Lupus Erythematosus, System; Ventricular Dysfunction, Left; Adolescent; Echocardiography/methods.

\section{Introduction}

Juvenile systemic lupus erythematosus (JSLE) is an inflammatory disease involving multiple organs and systems. The average age at onset is between 11 and 12 years old, approximately $80 \%$ of patients are female. ${ }^{1,2}$ It usually has a more severe clinical course raising morbidity and mortality rates. ${ }^{1-3}$ Cardiovascular complications occur in $50 \%$ of patients, and are the third leading cause of death, requiring special attention. ${ }^{3,4}$ They normally present heart failure (HF) and preserved ejection fraction (HFpEF), and initially manifest as subclinical diastolic dysfunction (DD). ${ }^{4}$ The early diagnosis of this dysfunction is fundamental, since at this early stage of DD, factors that interfere with the progression of HF can be modified. This recognition is a complex process that requires a systematized evaluation. Echocardiography is the mainstay of DD evaluation, as it is noninvasive and available. ${ }^{5,6}$ In adolescents, studies are scarce, and little is known about LV function in JSLE. ${ }^{7}$

Given the importance of the topic, this study used echocardiography to assess left ventricular (LV) diastolic function in JSLE, based on the hypothesis that the inflammatory process arising from pathogenesis and/or treatment would be associated with the occurrence of subclinical LV DD due to a complex interplay of factors leading to chronic inflammation, deposition of immune 
complexes, and chronic infiltrates that result in impaired LV relaxation and distensibility as early as adolescence. . $^{89}$

\section{Methods}

This was a cross-sectional, observational, control group study conducted with patients of the NESA-HUPE/UERJ, Rio de Janeiro, Brazil, from March 2017 to June 2019. Echocardiographic tests were performed at Lup Dup and Babycor Pediatric and Fetal Cardiology Clinics, for routine and/or sports practice evaluation.

Inclusion criteria were adolescents aged 10 to 20 years, diagnosed with JSLE, in follow-up at NESA-HUPE/UERJ, and healthy adolescents of the same age group, who had routine echocardiograms. Laboratory tests of the control group (complete blood count, lipid count, coagulogram, biochemistry, urea, creatinine, TSH and free T4 tests), echocardiogram and ECG were normal. The exclusion criteria were patients diagnosed with cardiac arrhythmia, congenital heart disease, systolic dysfunction, and inadequate echocardiographic window. The data were obtained from medical records that were reviewed by the same pediatric rheumatologist to obtain clinical, laboratory and therapeutic management data. Disease activity was determined according to the SLE Disease Activity Index 2000, revised in 2012 (SLEDAI-2K). ${ }^{4,10}$ A SEDAI-2K value $>4$ was defined as an indicator of disease activity. All patients completed a clinical questionnaire at the time of examination. ${ }^{10}$

Two-dimensional color transthoracic Doppler echocardiography, M-mode, with tissue Doppler was performed using a Philips HD 11 (Philips, Andover MA, USA) device and 3 to $5 \mathrm{MHz}$ transducers. The echocardiogram was performed in the left lateral decubitus position. All images were recorded. All echocardiographic examinations were performed and interpreted by the same observer and according to the recommendations of the American Society of Echocardiography. ${ }^{9}$ The echocardiographic variables studied were septal annular velocity $\mathrm{E}^{\prime}$ or lateral $\mathrm{E}^{\prime}$, mean E/E' ratio (lateral E' + septal E'/2), indexed left atrial volume (iVLA) by body surface area (BS), maximum reflux speed through the tricuspid (VT), mitral Doppler (E/A ratio), LV systolic diameter (LVDd), posterior wall in systole (PWs), aortic root.

For the purpose of identifying diastolic function, the 2016 recommendation cites four items: septal $\mathrm{E}^{\prime}$ speed less than or equal to $7 \mathrm{~cm} / \mathrm{sec}$. or lateral $\mathrm{E}^{\prime}$ $\leq 10 \mathrm{~cm} / \mathrm{m}$, mean $\mathrm{E} / \mathrm{E}^{\prime}$ ratio $>14$; reflux speed through the tricuspid $2.8 \mathrm{~m} / \mathrm{sec}$; and indexed LA volume $>34 \mathrm{ml} / \mathrm{m}^{2}$. If three or more items are negative, diastolic function is normal. If half is negative and half is positive, the diastolic function is indeterminate. If three or more items are positive, diastolic dysfunction is present. ${ }^{11}$ (Figure 1). Based on the diagnosis of JSLE, two groups were created: A- cases of JSLE (subdivided by disease activity, yes or no) and B- control group, composed of healthy adolescents and comparisons were made between the groups.

All Doppler measurements were averaged over 3 heartbeats, to minimize variations with breathing. Pulsed Doppler (PW) was used for accurate definition of ventricular interval times. ${ }^{12}$ Simultaneous electrocardiograms helped correlate flow timing with electrical changes.

\section{Indexed Left Atrial Volume}

The anteroposterior diameter of the left atrium was obtained by M-mode echo. Using two-dimensional echo, the indexed left atrial volume (iVLA) was gauged using the biplane Simpson technique on apical 4- and 2-chamber sections followed by indexing by body surface area. ${ }^{11-13}$ (Figure 2). The cut-off value indicating abnormality is iVLA $>34 \mathrm{ml} / \mathrm{m}^{2}{ }^{11}$

Tissue Doppler imaging of mitral annular velocities: septal and lateral

PW Tissue Doppler imaging (TDI) was performed on the apical section to acquire mitral annular velocities. (Figures: 3, 4 and 5) Measurements reflected the average of $\geq 3$ consecutive cardiac cycles.

E/A ratio; Pulsed wave (PW) Doppler was performed in the apical 4-chamber view to obtain mitral inflow velocities to assess LV filling. Color flow imaging was used for optimal Doppler beam alignment (Figure 6). ${ }^{11,13}$

\section{Tricuspid flow velocities}

The peak velocity of the tricuspid valve regurgitant jet was performed by positioning the sample volume in the reflux vena contracta. By continuous Doppler, the pulmonary systolic pressure was quantitatively estimated (Figures 7 and 8). ${ }^{11-14}$

Aortic root: Location of measurements performed were the aortic valve annulus (point of articulation of the aortic leaflets), the sinuses of Valsalva, the sinotubular junction, and the proximal ascending aorta. ${ }^{11-14}$ 


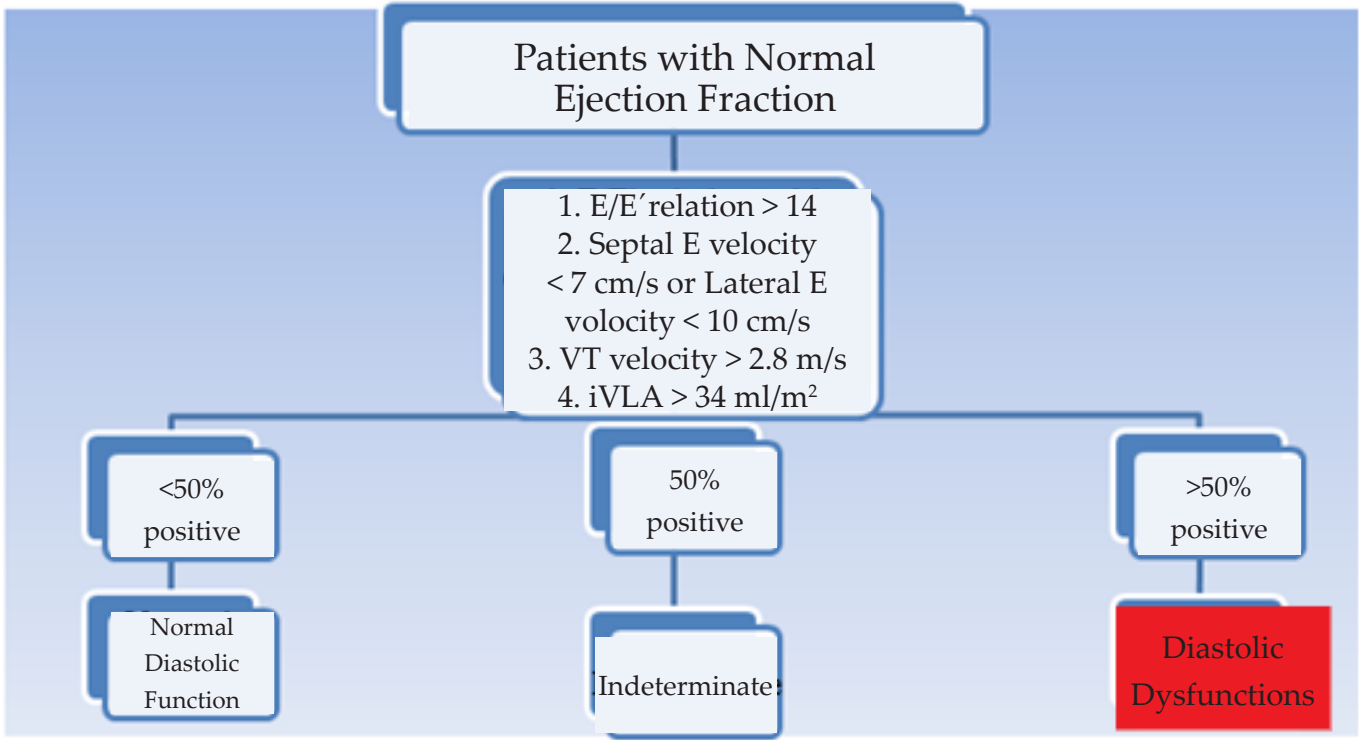

Figure 1 - Algorithm of diastolic function evaluation 2016.11

Source: prepared by the author. Based on the Journal of the American Society of Echocardiography 2016 29, 277-31411.

${ }^{*}(V T)$ velocity through the tricuspid; > 2.8 to $2.9 \mathrm{~m} / \mathrm{s}$, corresponding to *(PSAP) pulmonary artery systolic pressure of approximately $36 \mathrm{mmHg} .{ }^{11}$

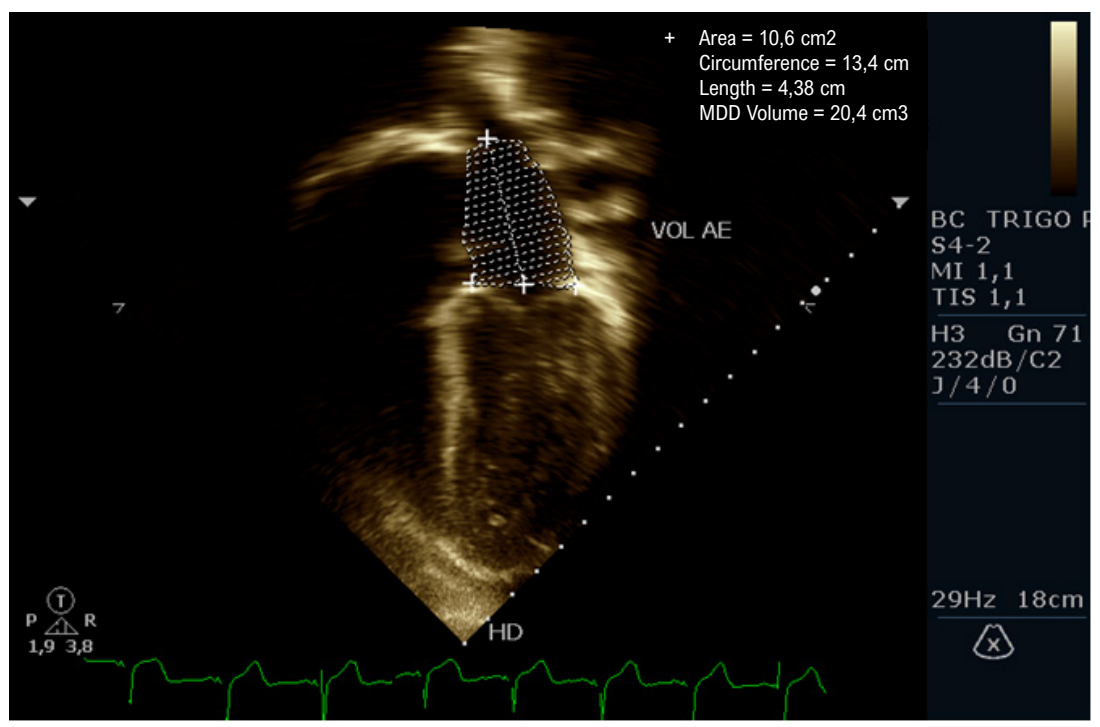

Figure 2 - Indexed volume of the left atrium. (iVLA)

Personal file

Planimetry of the area of the left atrium in the apical 4 chambers. 


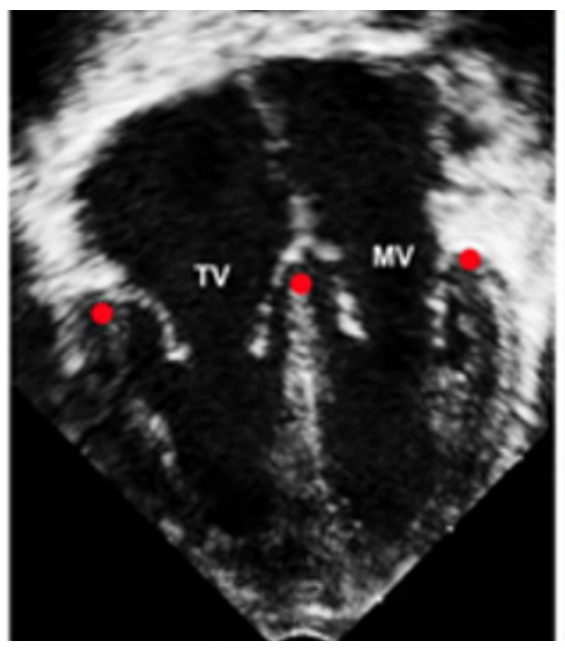

Figure 3 - Position of the sample volume in TDI and tissue Doppler evaluation at color Personal file

Nagueh et al. 201611
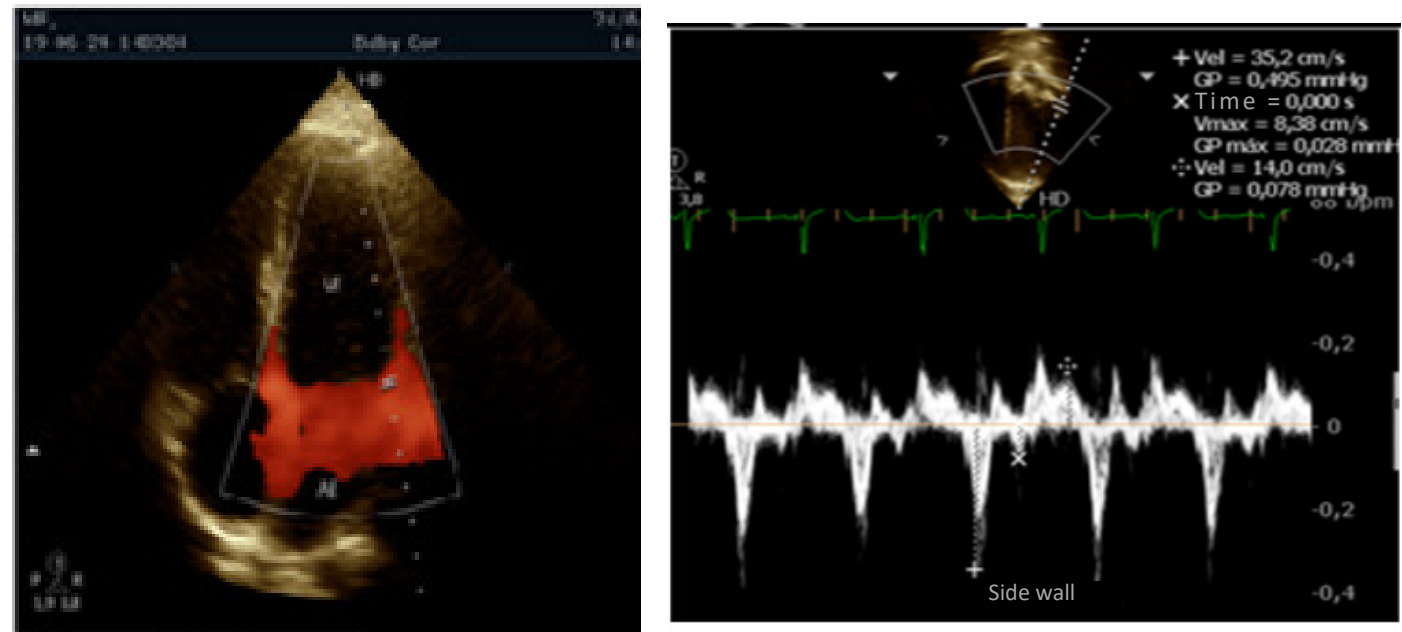

Figures 4 and 5 - Tissue Doppler, lateral E' wave. Pulsed Doppler analysis of mitral flow velocities. 


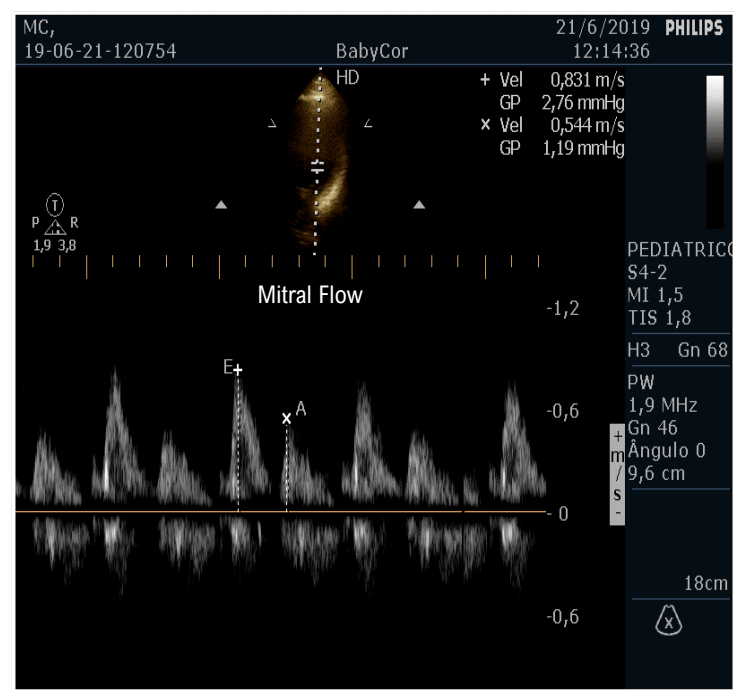

Figure 6 - Mitral valve Doppler

Personal file.

Normal mitral flow pattern acquired by PW Doppler. Mitral E velocity and A velocity
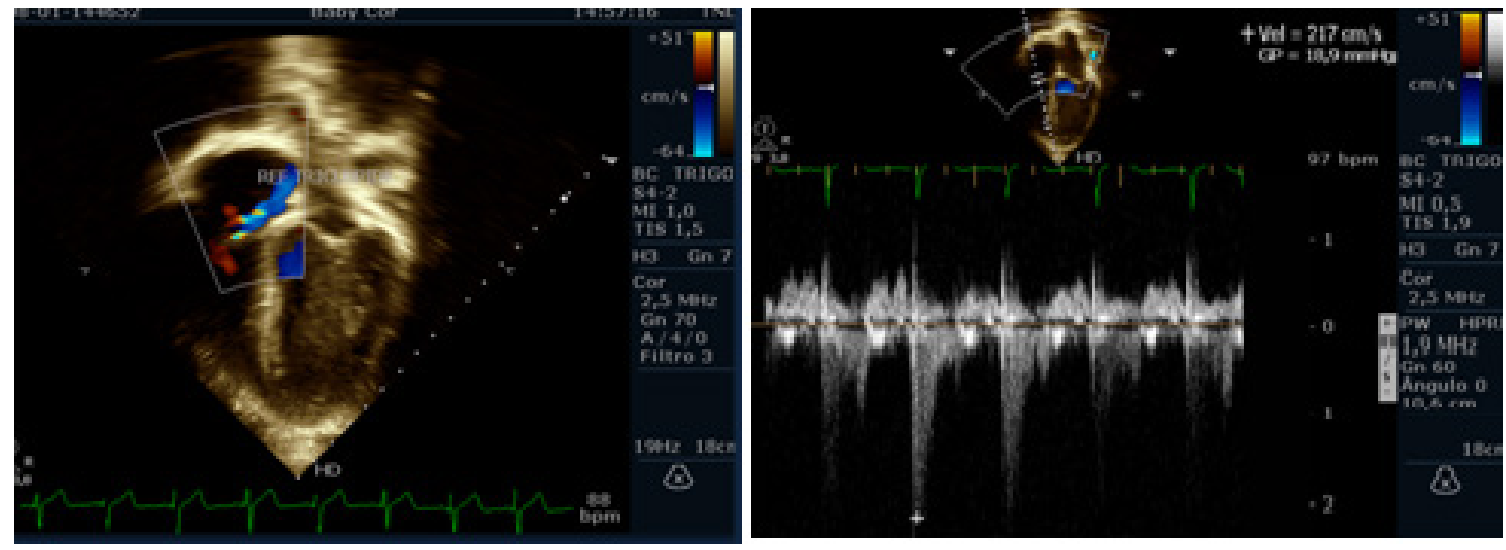

Figures 7 and 8 - Tricuspid flow velocities at color and tricuspid flow velocities at pulsed Doppler. Personal file.

Color Doppler of reflux through the tricuspid (left); Doppler of tricuspid regurgitant jet (right).

\section{Two-dimensional echocardiography}

The dimensions of the cardiac chamber were obtained by M-mode in the parasternal long axis section, guided by two-dimensional (2D) echocardiography following the recommendations of the American Society of Echocardiography and the European Association of Cardiovascular Imaging from March 2015. ${ }^{14,15}$ The dimensions of the left ventricle, interventricular septum, and posterior wall both at systole and diastole; aorta and left atrium were measured.

\section{Statistical analysis}

The Stata program version 13.0 (Stata Corp) was used for statistical analysis. A significance level of $5 \%(\mathrm{p}<0.05)$ was adopted for all analyses. The data did not show normal distribution (Shapiro Wilk test; $p$ value $=0.02$ ). In the bivariate analyses, Mann-Whitney and Kruskal-Wallis tests were used for continuous variables, and for categorical variables, Fisher's exact test was used. Continuous variables were presented 
Table 1 - Clinical characteristics and echocardiographic data according to the diagnosis of JSLE (n=98)

\begin{tabular}{|c|c|c|c|}
\hline \multirow{2}{*}{ Variable } & \multicolumn{2}{|c|}{ JSLE Diagnosis } & \multirow{2}{*}{ p value* } \\
\hline & Yes & No & \\
\hline Age (years) & $14(12-16)$ & $14(13-15)$ & 0.43 \\
\hline $\mathrm{BS}\left(\mathrm{m}^{2}\right)$ & $1.0(0.8-1.2)$ & $1.1(0.9-1.2)$ & 0.55 \\
\hline Females (\%) & 83.6 & 24.4 & $<0.001 \#$ \\
\hline Ind. Vol. Left Atrium & $15.7(12.7-18.9)$ & $12.2(10.0-15.0)$ & $<0.001$ \\
\hline Aortic root & $23.0(22.0-25.0)$ & $22.0(20.0-25.0)$ & 0.11 \\
\hline LA & $26.0(23.0-29.0)$ & $27.0(23.3-29.0)$ & 0.42 \\
\hline PWd & $10.0(9.0-11.5)$ & $10.0(8.5-12.0)$ & 0.87 \\
\hline Lv Systolic Diameter & $24.0(23.0-25.5)$ & $23.0(20.0-26.0)$ & 0.18 \\
\hline E' Septal line & 0.18 & $18.1(15.4-20.9)$ & 0.13 \\
\hline E' Lateral line & $24.7(20.1-32.7)$ & $28.3(23.6-35.2)$ & 0.01 \\
\hline E' Medium Septal line & $6.0(4.8-6.8)$ & $5.7(4.8-7.3)$ & 0.97 \\
\hline E' Lateral line & $3.9(3.3-4.6)$ & $3.5(3.2-4.7)$ & 0.30 \\
\hline E line & $4.7(3.8-5.4)$ & $4.3(4.0-5.2)$ & 0.42 \\
\hline $\mathrm{E}^{\prime} / \mathrm{A}$ ratio & $1.6(1.3-2.1)$ & $1.9(1.7-2.2)$ & 0.001 \\
\hline TV & $1.9(1.7-2.2)$ & & 0.20 \\
\hline
\end{tabular}

Table 2 - Echocardiographic findings in patients with JSLE according to disease activity (n=49)

\begin{tabular}{lccc}
\hline \multirow{2}{*}{ Variable } & \multicolumn{2}{c}{ Disease activity } & p value* \\
\cline { 2 - 4 } & Yes & No & 0.70 \\
\hline Ind. Vol. Left Atrium & $15.7(13.0-18.5)$ & $15.8(10.8-19.0)$ & 0.03 \\
Aortic root & $22.0(21.0-25.0)$ & $24.0(22.5-26.5)$ & 0.93 \\
LA & $26(24.0-28.0)$ & $26.0(22.0-29.0)$ & 0.69 \\
PWd & $10.0(9.0-11.5)$ & $10.0(8.8-11.2)$ & 0.55 \\
Lv Systolic diameter & $25.0(23.0-26.0)$ & $24.0(22.0-25.0)$ & 0.26 \\
E' Septal line & $16.4(13.4-18.8)$ & $17.9(15.9-20.1)$ & 0.15 \\
E' Lateral line & $23.6(18.7-29.1)$ & $29.2(22.0-34.7)$ & 0.74 \\
E' Medium Septal Line & $6.0(4.8-6.8)$ & $5.7(4.8-7.3)$ & 0.35 \\
E' Medium Lateral line & $4.0(3.3-4.8)$ & $3.6(2.8-4.4)$ & 0.60 \\
E line & $4.8(4.0-5.6)$ & $4.5(3.7-5.4)$ & 0.21 \\
E'/A ratio & $1.5(1.3-2.0)$ & $1.7(1.4-2.2)$ & 0.18 \\
TV & $1.9(1.7-2.2)$ & $1.8(1.5-2.1)$ & \\
\hline
\end{tabular}


Table 3 - Echocardiographic parameters in patients with JSLE, according to corticosteroid use ( $\mathrm{n}=49$ )

\begin{tabular}{|c|c|c|c|}
\hline \multirow{2}{*}{ Variable } & \multicolumn{2}{|c|}{ Corticosteroid use } & \multirow{2}{*}{ p value* } \\
\hline & (yes) & (no) & \\
\hline Age (years) & $14(12-16)$ & $15(13-17)$ & 0.13 \\
\hline $\mathrm{BS}\left(\mathrm{m}^{2}\right)$ & $1.07(1-1.23)$ & $1.05(0.94-1.21)$ & 0.38 \\
\hline Ind. Vol. Left Atrium & $15.23(12.69-18.5)$ & $16(12.7-19.2)$ & 0.93 \\
\hline Aortic root & $23(22-26)$ & $23(22-25)$ & 0.67 \\
\hline LA & $26.75(24-29)$ & $25(22.7-28)$ & 0.31 \\
\hline PWd & $10(9-11.5)$ & $10(8.5-11.5)$ & 0.57 \\
\hline Lv Systolic diameter & $24(23-26)$ & $24(23-25)$ & 0.66 \\
\hline E' Septal line & $15.75(13.2-18.4)$ & $17.9(16.2-20.4)$ & 0.03 \\
\hline$E^{\prime}$ Lateral line & $22.3(17-30.6)$ & $25.5(22-33.5)$ & 0.12 \\
\hline E' Medium Septal Line & $6.38(5.13-7.54)$ & $5.55(4.57-6.42)$ & 0.09 \\
\hline$E^{\prime}$ Medium Lateral line & $4.31(3.37-5.44)$ & $3.7(2.81-4.27)$ & 0.18 \\
\hline E line & $5.18(4.38-6.0)$ & $4.38(3.59-5.0)$ & 0.05 \\
\hline $\mathrm{E}^{\prime} / \mathrm{A}$ ratio & $1.52(1.31-2.19)$ & $1.72(1.33-2.18)$ & 0.71 \\
\hline TV & $21.35(17-28.5)$ & $20(13-22.5)$ & 0.12 \\
\hline
\end{tabular}

Table 4 - Logistic regression model for indexed Vol LA and variables (n=98)

\begin{tabular}{lcccc}
\hline Variable & OR & IC 95\% & Standard Error & p value \\
\hline JSLE & 3.3 & $1.10-9.9$ & 1.86 & 0.03 \\
\hline Model adjusted by sex. & & & & \\
\hline
\end{tabular}

by medians and interquartile range (IQ). Categorical variables were presented by frequencies and 95\% confidence intervals ( $95 \% \mathrm{CI}$ ). Multivariate logistic models were constructed to test echocardiographic parameters and possible associations. All variables with $p<0.20$ in the bivariate analysis were tested in the model.

This research was approved by the Research Ethics Committee (Opinion number 2.385.087). Informed consent was obtained from legal guardians as well as from the patients themselves.

\section{Results}

Ninety-eight patients were included. Of these, 49 patients diagnosed with JSLE and 49 healthy and asymptomatic adolescents. All patients had normal ejection fraction. Based on the 2016 guideline, no diastolic dysfunction was found in adolescents with JSLE. The median age was 14 years (IIQ 12-16) and body mass index (BMI) 21.2 (IIQ 19.3-23.6). The clinical characteristics and echocardiographic parameters of 
the study sample revealed a female in those diagnosed with JSLE, larger index volume of the left atrium, smaller lateral $\mathrm{E}^{\prime}$ and lower E/A ratio (Table 1).

Among adolescents with JSLE 44 (89.7\%) had a history of disease of less than 5 years, and 33 children (67.34\%) were in disease activity. Comparisons were made among patients diagnosed with JSLE according to disease activity. In those with disease activity, less aortic root was observed (Table 2).

Comparisons were made between echocardiographic parameters in patients with JSLE taking or not taking corticosteroids. In those taking this medication, lower septal E's were observed (Table 3).

To construct logistic regression models, echocardiographic parameters with statistical significance were categorized and distributed according to the $50^{\text {th }}$ percentile of the control group. Three models were calculated: Ind. Vol. LA (> 12), E' lateral line (> $28)$, and $E^{\prime} / A$ ratio (>1.9). The only model that revealed statistical significance was the variable JSLE as a predictor of higher indexed Vol LA (Table 4).

The regression model for the variable $\mathrm{E}$ lateral line did not result in any predictor variable with statistical significance. The regression model of the variable E'/A ratio resulted in a marginal result for the variable JSLE (OR 0.36; p value 0.05).

Regarding the use of hydroxychloroquine, only 2 $(4.08 \%)$ of patients with JSLE did not use it during the study period.

\section{Discussion}

The main result found in this study was the presence of normal diastolic function according to the criteria of the 2016 guideline. However, when compared to normal adolescents, there was a significant difference in some parameters of diastolic function assessment. The presence of JSLE influenced the following parameters: index volume of the larger LA, smaller lateral line $\mathrm{E}^{\prime}$ and smaller septal $E^{\prime}$, due to the influence of corticoid use, smaller E'/A ratio, and finally, lower aortic root values were observed in patients who were active. The sample size of this study, the short time of disease and follow-up may have interfered with this result.

This analysis of cardiac function by echocardiography enabled early evaluation of the heart of patients with JSLE with normal ejection fraction. JSLE patients had worse indices than controls, even though they were within normal values. Thus, this study highlights the need for monitoring diastolic function after the diagnosis of JSLE. In a study that evaluated function, Petri et al., ${ }^{4}$ showed that 4 to $71 \%$ of adults with lupus have some degree of diastolic dysfunction, with few or no associated comorbidities. ${ }^{4}$

In this casuistry, patients with JSLE had higher indexed LA volume values. In 2017, Singh et al., ${ }^{16}$ reinforced that LA strain could be used to detect diastolic dysfunction. ${ }^{16}$ Assessment of left atrial function provides important information related to diastolic dysfunction, as increased LA is usually associated with increased LV stiffness. In this study, the values were higher in subjects with JSLE regardless of disease activity or not, and in multivariate analysis the presence of JSLE was associated with higher values of indexed LA volume. The main role of the left atrium is to modulate left ventricular filling through adaptive changes in its mechanics and structure in the various LV filling patterns. Chronic or prolonged elevation of left atrial pressure may also be associated with natriuretic peptide elevation, left atrial remodeling, and increased risk of death. ${ }^{14}$ Currently, three-dimensional echo (3D-E) and myocardial strain imaging are being used promisingly for LA volume assessment, as they correlate well with cardiac computed tomography and nuclear magnetic resonance imaging. ${ }^{17}$

Regarding the E' lateral line, patients with JSLE had a lower value. The lower values are justified because the lateral line E' parameter is used to identify and measure the speed of myocardial movement, and this movement can be slowed down or delayed in the setting of dysfunction. As a result, the restoring forces in the myocardium increase the stretching load and tend to compensate for the deficit through compensatory mechanisms. Thus, lateral line E' wave analysis is a relatively insensitive measure of LV preload and has been shown to be useful in predicting higher LV filling pressure.

Regarding the E'/A ratio patients with JSLE showed lower values when compared to controls. The diagnosis of JSLE was not a predictor of lower E/A ratio values. Marginal statistical significance was observed. In this case, the sample size may have influenced the result; had the sample been a little larger, this index might have obtained statistical relevance. Lower E'/A ratio occurs when the LV presents progressing diastolic dysfunction. ${ }^{14}$ Impaired relaxation with normal LA size would ideally fall under diastolic dysfunction, but under the new scheme the classification would be 
considered indeterminate in the absence of an elevation in peak RT velocity. ${ }^{14,18}$ Therefore, it is possible that the updated 2016 guideline for the assessment of diastolic function creates a situation where diastolic dysfunction may be underdiagnosed.

Although no association between JSLE and aortic root has been reported in the literature, lower aortic root values have been observed for patients with JSLE in disease activity. This can be understood as higher aortic root stiffness representing structural and functional changes of the vessel wall caused by vasculitis. ${ }^{17,19}$ The replacement of the elastic band by the stiffer fibrous scar during the repair process, and the induction of metalloproteinases by inflammatory mediators may be cited as causes. ${ }^{20}$ Thus, although there is no description of the relationship between echocardiographic measurements of the aortic root and JSLE, it is suggested that aortic elasticity indices should be emphasized in patients. Persistent low-grade inflammation and endothelial dysfunction, which are interrelated, may also explain, at least in part, the JSLE-related increased arterial stiffness. ${ }^{20,21}$ Increased arterial stiffness increases the risk of cardiovascular disease by increasing blood pressure, increasing ventricular hypertrophy, decreasing coronary perfusion, and increasing the risk of stroke. ${ }^{22,23}$ Arterial function in adolescents and young adults with JSLE has not been studied to date and should be the subject of future research.

Limitations of this study are the short follow-up time, the sample size, and the absence of sequential examinations for evolutionary comparison. We suggest that the parameters of the EACVI 2016 guideline $^{11}$ should be used along with those of the EACVI 2009 guideline ${ }^{15}$ in a thorough and systematic manner, at the very first moment of evaluation, as they are important for the assessment of ventricular diastolic function. Assessment of diastolic function in JSLE is a recognized prognostic severity marker, and changes may be early and subclinical. We suggest that echocardiography be performed early in these patients.

\section{References}

1. Hedrich CM, Zappel H, Straub S, Laass MW, Wieczorek K, Hahn G, et al. Early onset systemic lupus erythematosus: differential diagnoses, clinical presentation, and treatment options. Clin Rheumatol. 2011;30(2): 275-83. Doi:10.1007/s10067-010-1576-2.

2. Sztajnbok FR, Serra CR, Rodrigues MC, Mendoza E. Doenças reumáticas na adolescência [Rheumatic diseases in adolescence]. J Pediatr (Rio J). 2001; 77(2): S234-44. Doi:10.2223/jped.311.

\section{Conclusions}

No changes in FD following EACVI guidelines were detected in adolescents with JSLE and ejection fraction was normal. However, among patients with JSLE, there was a higher prevalence of higher indexed LA volume, lower lateral E' values and lower E'/A ratio. Aortic root measurements were smaller in patients with active JSLE.

\section{Author contributions}

Acquisition of data: Loureiro TN. Analysis and interpretation of the data: Loureiro TN. Statistical analysis: Valete COS. Writing of the manuscript: Loureiro TN. Critical revision of the manuscript for intellectual content: Sztajnbok FR e Castier MB.

\section{Potential Conflict of Interest}

No potential conflict of interest relevant to this article was reported.

\section{Sources of Funding}

There were no external funding sources for this study.

\section{Study Association}

This article is part of the thesis of master submitted by Talita Nolasco Loureiro, from Universidade do Estado do Rio de Janeiro.

\section{Ethics approval and consent to participate}

This study was approved by the Ethics Committee of the Hospital Universitário Pedro Ernesto/ Universidade do Estado do Rio de Janeiro under the protocol number 2.385.087. All the procedures in this study were in accordance with the 1975 Helsinki Declaration, updated in 2013. Informed consent was obtained from all participants included in the study.

3. Harry O, Yasin S, Brunner H. Childhood-Onset Systemic Lupus Erythematosus: A Review and Update. J Pediatr. 2018; 196: 22-30.e2. Doi:10.1016/j.jpeds.2018.01.045.

4. Petri M, Orbai AM, Alarcón GS, Gordon C, Merrill JT, Fortin PR, et al Derivation and validation of the Systemic Lupus International Collaborating Clinics classification criteria for systemic lupus erythematosus. Arthritis Rheum. 2012; 64(8):2677-86. Doi:10.1002/art.34473. 
5. Tucker LB, Uribe AG, Fernández M, Vilá LM, McGwin G, Apte M, et al. Adolescent onset of lupus results in more aggressive disease and worse outcomes: results of a nested matched case-control study within LUMINA, a multiethnic US cohort (LUMINA LVII). Lupus. 2008; 17(4), 314-22. Doi:10.1177/0961203307087875.

6. Brown AC. Lupus erythematosus and nutrition: a review of the literature. J Ren Nutr. 2000; 10(4):170-83. Doi:10.1053/jren.2000.16323.

7. Moder KG, Miller TD, Tazelaar HD. Cardiac involvement in systemic lupus erythematosus. Mayo Clin Proc. 1999; 74(3):275-84 Doi:10.4065/74.3.275 .

8. Winslow TM, Ossipov MA, Fazio GP, Foster E, Simonson JS, Schiller NB. The left ventricle in systemic lupus erythematosus: initial observations and a five-year follow-up in a university medical center population. Am Heart J. 1993; 125(4):1117-22. Doi:10.1016/0002-8703(93)90123-q.

9. Gladman D, Ginzler E, Goldsmith C, Fortin P, Liang M, Urowitz M, et al. The development and initial validation of the Systemic Lupus International Collaborating Clinics/American College of Rheumatology damage index for systemic lupus erythematosus. Arthritis Rheum. 1996; 39(3):363-9. Doi:10.1002/art.1780390303.

10. Fonseca AR, Gaspar-Elsas MI, Land MG, Oliveira SK. Comparison between three systems of classification criteria in juvenile systemic lupus erythematous. Rheumatology (Oxford). 2015; 54(2): 241-7. Doi:10.1093/ rheumatology/keu278.

11. Nagueh SF, Smiseth OA, Appleton CP, Byrd BF, Dokainish H, Edvardsen $\mathrm{T}$, et al. Recommendations for the Evaluation of Left Ventricular Diastolic Function by Echocardiography: An Update from the American Society of Echocardiography and the European Association of Cardiovascular Imaging. Eur Heart J Cardiovasc Imaging. 2016; 17(12): 1321-60. Doi:10.1093/ehjci/jew082.

12. Oktay AA, Rich JD, Shah SJ. The emerging epidemic of heart failure with preserved ejection fraction. Curr Heart Fail Rep. 2013;10(4):401-10. Doi:10.1007/s11897-013-0155-7.

13. Lopez L, Colan SD, Frommelt PC, Ensing GJ, Kendall K, Younoszai AK, et al. Recommendations for quantification methods during the performance of a pediatric echocardiogram: a report from the Pediatric Measurements Writing Group of the American Society of Echocardiography Pediatric and Congenital Heart Disease Council. J Am Soc Echocardiogr. 2010; 23(5):465-95. Doi:10.1016/j.echo.2010.03.019.
14. Lang RM, Badano LP, Mor-Avi V, Afilalo J, Armstrong A, Ernande $\mathrm{L}$, et al. Recommendations for cardiac chamber quantification by echocardiography in adults: an update from the American Society of Echocardiography and the European Association of Cardiovascular Imaging. J Am Soc Echocardiogr. 2015; 28(1):1-39. e14. Doi: 10.1016/j. echo.2014.10.003.

15. Nagueh SF, Appleton CP, Gillebert TC, Marino PN, Oh JK, Smiseth OA, et al. Recommendations for the evaluation of left ventricular diastolic function by echocardiography. European journal of echocardiography: the journal of the Working Group on Echocardiography of the European Society of Cardiology. 2009; 10(2):165-93. Doi:10.1093/ejechocard/jep007.

16. Singh A, Addetia K, Maffessanti F, Mor-Avi V, Lang RM. LA Strain for Categorization of LV Diastolic Dysfunction. JACC. Cardiovasc Imaging. 2017; 10(7):735-43. Doi:10.1016/j.jcmg.2016.08.014

17. Otto KM, Catherine M, Schwaegler, Rebeca G. The practice of clinical echocardiography. $5^{\text {th }}$ ed Philadelphia:Elsevier; 2016.

18. Urowitz MB, Bookman AA, Koehler BE, Gordon DA, Smythe HA, Ogryzlo MA. The bimodal mortality pattern of systemic lupus erythematosus. Am J Med. 1976; 60(2): 221-5. Doi:10.1016/0002-9343(76)90431-9.

19. Cheung YF, Brogan PA, Pilla CB, Dillon MJ, Redington AN. Arterial distensibility in children and teenagers: normal evolution and the effect of childhood vasculitis. Arch Dis Child. 2002; 87(4):348-51. Doi:10.1136/ adc.87.4.348.

20. Boros CA, Bradley TJ, Cheung MM, Bargman JM, Russell JL, McCrindle BW, et al. Early determinants of atherosclerosis in paediatric systemic lupus erythematosus. Clin Exp Rheumatol. 2011; 29(3): 575-81.

21. Tomiyama H, Hashimoto H, Hirayama $\mathrm{Y}$, Yambe M, Yamada J, Koji $\mathrm{Y}$, et al. Synergistic acceleration of arterial stiffening in the presence of raised blood pressure and raised plasma glucose. Hypertension. 2006; 47(2):180-8. Doi: 10.1161/01.HYP.0000198539.34501.1a

22. Safar ME, Thomas F, Blacher J, Nzietchueng R, Bureau JM, Pannier B, et al. Metabolic syndrome and age-related progression of aortic stiffness. J Am Coll Cardiol. 2006; 47(1):72-5. Doi:10.1016/j.jacc.2005.08.052.

23. Burkhoff D, Mirsky I, Suga H. Assessment of systolic and diastolic ventricular properties via pressure-volume analysis: a guide for clinical, translational, and basic researchers. Am J Physiol Heart Circ Physiol. 2005; 289(2):H501-H512. Doi:10.1152/ajpheart.00138.2005 